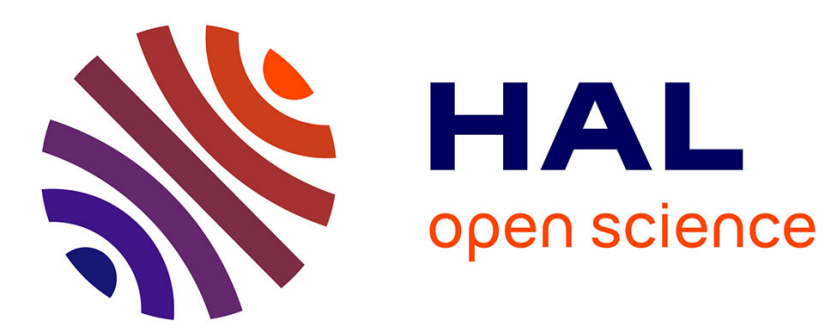

\title{
Extension of the bifurcation method for diffusion coefficients to porous medium transport
}

Cédric Descamps, Gerard L. Vignoles

\section{To cite this version:}

Cédric Descamps, Gerard L. Vignoles. Extension of the bifurcation method for diffusion coefficients to porous medium transport. Comptes rendus de l'Académie des sciences. Série IIb, Mécanique, 2000, 328 (6), pp.465-470. 10.1016/S1620-7742(00)00038-6 . hal-00327610

\section{HAL Id: hal-00327610 \\ https://hal.science/hal-00327610}

Submitted on 16 Oct 2008

HAL is a multi-disciplinary open access archive for the deposit and dissemination of scientific research documents, whether they are published or not. The documents may come from teaching and research institutions in France or abroad, or from public or private research centers.
L'archive ouverte pluridisciplinaire HAL, est destinée au dépôt et à la diffusion de documents scientifiques de niveau recherche, publiés ou non, émanant des établissements d'enseignement et de recherche français ou étrangers, des laboratoires publics ou privés. 


\title{
Extension of the bifurcation method for diffusion coefficients to porous medium transport
}

\author{
C. DESCAMPS \\ Snecma Propulsion Solide \\ Les 5 chemins, \\ F33187 LE HAILLAN Cedex, France \\ Cedric.descamps@snecma.fr \\ and \\ G. L. VIGNOLES

\section{LCTS} \\ Laboratoire des Composites ThermoStructuraux \\ 3,Allée La Boëtie - Université Bordeaux 1 \\ F33600 PESSAC, France \\ vinhola@1cts.u-bordeaux1.fr
}

Published in :

C. R. Acad. Sci. série IIb (mécanique) vol. 328 (2000), pp. 465-470. 


\title{
Extension of the bifurcation method for diffusion coefficients to porous medium transport
}

\author{
Cédric Descamps and Gérard L. Vignoles \\ Laboratoire des Composites ThermoStructuraux \\ (UMR 5801 CNRS-SNECMA-CEA-UB1) \\ 3, Allée La Boëtie, Domaine Universitaire, \\ F33600 PESSAC, France \\ Fax : (00 33) 556841225 \\ E-mail : descamps@lcts.u-bordeaux.fr
}

\begin{abstract}
We present an extension of Bartlett's bifurcation method (Bartlett et al. (1968)) for the approximate computation of multicomponent diffusion coefficients in a gaseous mixture to diffusion in porous media. On behalf of the remark that the bifurcation coefficients $F_{i}$ are merely proportional to the square root of the molar masses $M_{i}$, we state that Knudsen diffusion may also be represented through some bifurcation factor $F_{K}$. This approximation is tested in a variety of cases, displaying good results except for very light gas species.
\end{abstract}

\section{Extension de la méthode de bifurcation pour les coeffi- cients de diffusion au transport en milieu poreux}

Résumé - Nous présentons une extension de la méthode de bifurcation de Bartlett et al. (1968) pour le calcul approché des coefficients de diffusion multicomposants 
dans un mélange gazeux à la diffusion en milieu poreux. Partant de la constatation que les coefficients de bifurcation $F_{i}$ sont approximativement proportionnels à la racine carrée des masses molaires $M_{i}$, il est posé que la diffusion de Knudsen peut aussi être représentée par un facteur de bifurcation $F_{K}$. Cette approximation est testée pour différents cas, et montre de bons résultats sauf pour des espèces très légères.

Mots-clés: Diffusion multicomposants; Milieux poreux; Méthode d'approximation

Key words: Multicomponent diffusion; Porous Media; Approximation method

\section{Version française abrégée}

Pour le traitement de la diffusion gazeuse multicomposants, qui requiert une inversion des relations de Stefan-Maxwell (éq. 1), Bartlett et al. (1968) ont proposé une méthode dite "de bifurcation", basée sur la constatation que chaque coefficient de diffusion binaire pouvait être réécrit suivant l'équation (4). Ceci est justifié en fait par la corrélation assez étroite entre les facteurs de bifurcation $F_{i}$ et l'inverse de la racine carrée des masses molaires $M_{i}^{-1 / 2}$. Une expression explicite pour les flux massiques est alors obtenue en fonction des gradients (éqs. 5,6). Les coefficients de bifurcation doivent être pré-calculés par une méthode de minimisation d'erreur, comme le simplexe.

Nous proposons d'étendre cette procédure aux équations de gaz poussiéreux de Mason et Malinauskas (1983) (eqs. 10), qui contiennent en particulier la contribution supplémentaire de la diffusion de Knudsen (éq. 12). Comme celleci fait aussi intervenir une proportionnalité à $M_{i}^{-1 / 2}$, il est donc possible de 
garder les mêmes $F_{i}$ pour les gaz et d'introduire un facteur $F_{K}$ pour le milieu poreux (éq. 13). Des relations explicites sont alors obtenues pour les flux massiques diffusifs des gaz (éqs. 21,22), et en prenant correctement en compte les dépendances différentes des diffusions binaire et de Knudsen à la température et à la pression, on doit corriger $F_{K}$ suivant l'expression (24).

La méthode originalement proposée présente une précision de l'ordre de quelques pour-cents, sauf pour des espèces très légères ou très lourdes. C'est également le cas de l'extension proposée ici.

\section{Introduction}

In many modeling problems involving gas mixtures (distillation, combustion, catalysis, etc ...), an important question is how to treat multicomponent diffusion. Conceptual difficulties have been overcome since a long time, but numerical issues are still of actuality, because achieving simultaneously accuracy, convergence and computational rapidity is not a simple task. In this context, the bifurcation method appeared as a fairly good compromise. On the other hand, a popular treatment of diffusive fluxes in porous media, featuring Knudsen diffusion (also called effusion), is the Dusty-Gas Model, which requires as much computational effort as for the determination of free-medium diffusive fluxes. We address here an extension of the bifurcation principle to gases in porous media. In a first part, the principle of the bifurcation method 
will be recalled, as well as some results on its accuracy ; then, the extension to the Dusty-Gas Model frame will be presented and tested.

\section{The Bifurcation Method}

Let us first recall briefly the context of Bartlett's approximation.

\subsection{Multicomponent diffusion relations}

Molar diffusive fluxes $J_{i}$ are related to the respective forces $\nabla P_{i} / \mathcal{R} T$ through Maxwell-Stefan relations :

$$
-\frac{\nabla P_{i}}{\mathcal{R} T}=\sum_{j \neq i}^{n} \frac{x_{j} J_{i}-x_{i} J_{j}}{\mathcal{D}_{i j}}
$$

In a mass balance equation, the divergence of the fluxes are needed : this means that eq. (1) has to be solved for the fluxes. This system also presents also a singularity because (i) all mass fluxes have to sum up to zero and (ii) all gradients have to sum up to $\nabla P_{\text {tot }} / \mathcal{R} T$, which is determined elsewhere. Removing these singularities and solving for the fluxes may lead to heavy computations, so a direct, explicit formula would be of great practical interest in the case of large systems e.g. combustion or pyrolysis problems (Giovangigli (1996))

From Chapman and Enskog's theory of gases (Reid et al. (1987), Bird et al. 
(1960)), the pair diffusion coefficients $\mathcal{D}_{i j}$ read :

$$
\mathcal{D}_{i j}=\frac{3}{16} \frac{\sqrt{4 \pi \mathcal{R} T}}{\pi \sqrt{M_{i j}} \sigma_{i j}^{2} \Omega_{i j}^{D}}
$$

where $M_{i j}$ is the reduced mass of the pair $(i, j)$ :

$$
M_{i j}=\frac{1}{2} \frac{M_{i} M_{j}}{M_{i}+M_{j}}
$$

\subsection{Principle of the bifurcation method}

The principle of the bifurcation method is to state that each multicomponent diffusion coefficient may be expressed as :

$$
\mathcal{D}_{i j}=\frac{D^{r e f}}{F_{i} F_{j}}
$$

where $D^{r e f}$ is some reference diffusion coefficient and $F_{i}$ a bifurcation factor proper to each species $i$. If $n$ species are present, then only $n$ factors $F_{i}$ are to be determined instead of $n(n-1) / 2$ coefficients $\mathcal{D}_{i j}$. In addition to this, a direct, explicit expression for the diffusive mass fluxes as a function of the gradients is obtained :

$$
j_{i}=M_{i} J_{i}=-\sum_{j=1}^{n} D_{i j} \nabla P_{j}
$$


where the multicomponent diffusion coefficients read :

$$
\left\{\begin{array}{l}
D_{i j}=-\omega_{i} \frac{M_{j}}{\mathcal{R} T F_{j}} \frac{D^{r e f}}{\sum_{l=1}^{n} x_{l} F_{l}} \quad \text { si } i \neq j \\
D_{i i}=\left(1-\omega_{i}\right) \frac{M_{i}}{\mathcal{R} T F_{i}} \frac{D^{r e f}}{\sum_{l=1}^{n} x_{l} F_{l}} \text { si } i=j
\end{array}\right.
$$

The reader is referred to the original reference (Bartlett et al. (1968)) for the detailed derivation of the latter expressions. Using this relation speeds up strongly the resolution of mass transport problems, while keeping the main physical constraint that the sum of the mass fluxes is zero.

Comparing (4) to the theoretical expression (2), one sees that this approximation will be valid as soon as :

$$
\frac{\sqrt{M_{i}+M_{j}}}{\sigma_{i j}^{2} \Omega_{i j}^{D}}=c s t=G
$$

The quantity $G$ in this relation has been computed for a set of 69 gas species, that is, for 2346 species pairs. Fig. 1 is an histogram of $G$, showing that this quantity possesses a modest standard deviation. The most important deviations were observed for very heavy, poorly volatile species such as $\mathrm{HgI}_{2}$. In order to determine the $F_{i}$ coefficient set, the simplex method is used. It is chosen to minimize the following error function :

$$
E=\sum_{i=1}^{n} \sum_{j=i+1}^{n}\left(F_{i} F_{j} \mathcal{D}_{i j}-D^{r e f}\right)^{2}
$$


It has been checked that the results obtained in the case of a 3-species set are exactly equal to the analytical prediction, since in this case the approximation is indeed exact.

Bartlett et al. (1968) report from their tests a mean error of $5 \%$ on the multicomponent diffusion coefficients. We also performed a test on a set of 14 hydrocarbons plus molecular hydrogen. The mean error is $2.03 \%$. The maximal deviation is $25.75 \%$ for the $H_{2}-C_{4}$ pair. This was to be expected since they are the lightest species present in the system : their $G$ factor is well below the mean $G$ factor of all species pairs. For the 69 -species system used to check validity of relation (7), the mean error was $3.44 \%$, showing reasonable usefulness of the approximation scheme to large systems.

\subsection{Influence of temperature}

As the binary diffusion coefficients vary with temperature in a moderate but complicated way (from $T^{3 / 2}$ to $T^{2}$ ), one should a priori take into account a thermal variation of the $F_{i}$ factors. Fig. 2 shows that when $T$ increases, the $F_{i}$ concerning the lightest species tend to increase while the heaviest species factors tend to decrease. However, one notices that the overall thermal variation in a broad temperature range $(300-1500 \mathrm{~K})$ for the $F_{i}$ is small compared to the initial errors due to the method. Accordingly, one may safely consider that these factors do not vary independently with temperature, the global dependence of diffusion on $T$ being incorporated inside $D^{r e f}$. 


\subsection{Correlations with molar masses}

Bartlett et al. (1968) also has shown from experimental data of Svehla (1962) that the $F_{i}$ may be estimated from the empirical correlation :

$$
F_{i} \propto M_{i}^{0.461}
$$

This fact may be expected from formulas $(2,3)$, where it is readily seen that $\mathcal{D}_{i j}$ is proportional to $\left(M_{i} M_{j}\right)^{-1 / 2}$ if the $G$ factor in eq. (1) does not vary appreciably. Fig. 4 shows a plot of the $F_{i}$ computed in the 69 -species test case versus $M_{i}^{-1 / 2}$, in which direct proportionality appears as a fairly good approximation.

\section{Application to diffusion in porous media}

\subsection{The Dusty-Gas Model equations}

A popular extension of the Stefan-Maxwell relations to porous media is the

Dusty-Gas Model (Mason and Malinauskas (1983)). Knudsen diffusion is viewed as the diffusion of a special gas pair, namely a true gas and the solid phase described as a collection of giant gas molecules ("dust") which are held fixed with respect to the laboratory referential. This "dust" plays the role of an 
$(n+1)^{t h}$ species. Relations (1) become then :

$$
\sum_{j \neq i}^{n} \mathcal{D}_{i j, \mathrm{eff}}^{-1}\left(x_{i} \vec{J}_{j}^{\mathcal{D}}-x_{j} \vec{J}_{i}^{\mathcal{D}}\right)-D_{i K, \mathrm{eff}}^{-1} \vec{J}_{i}^{\mathcal{D}}=\frac{\nabla P_{i}}{\mathcal{R} T}
$$

where the gas pair diffusion coefficients have been replaced by effective quantities $\mathcal{D}_{i j, \text { eff }}$ which take into account the influence of the porous medium :

$$
\mathcal{D}_{i j, \mathrm{eff}}=\epsilon \eta_{b}^{-1} \mathcal{D}_{i j}
$$

and where the Knudsen diffusion coefficients $D_{i K \text {,eff }}$ read :

$$
D_{i K, \mathrm{eff}}=\epsilon \eta_{K}^{-1} D_{i K}=\epsilon \eta_{K}^{-1} \frac{1}{3} \sqrt{\frac{8 k_{B} T}{\pi M_{i}}} \overline{d_{h}}
$$

The quantities $\epsilon, \overline{d_{h}}, \eta_{b}$, and $\eta_{K}$ refer respectively to the porosity, mean pore diameter, binary diffusion tortuosity, and Knudsen diffusion tortuosity for the considered porous medium. All of them may be coordinate-dependent if the medium is non-homogeneous, and the last two are second-order symmetric tensors in the case of an anisotropic medium (Ofori and Sotirchos (1997)). In eq. 12 we see clearly that the proportionality to $M_{i}^{-1 / 2}$ is preserved for the true gas $i$. Accordingly, the same kind of $F_{i}$ that is used for multicomponent diffusion may be used for the approximation of Knudsen diffusion. 


\subsection{Applying the bifurcation method}

Relation (12) may be rewritten :

$$
D_{i K}=\frac{D^{r e f}}{F_{i} F_{K}}
$$

where $F_{i}$ et $D^{r e f}$ are the diffusion factors and the reference diffusivity computed from the binary(ordinary) diffusion data. $F_{K}$ incorporates a contribution arising from the solid phase. As a matter of fact, $F_{K}$ should be considered as a field when it is non-homogeneous.

Now direct explicit relations for the fluxes may be attained, as in the case of standard multicomponent diffusion. Summing up eqs. (10) on all gas species $i$, one has :

$$
\sum_{i=1}^{n}\left(\sum_{j \neq i}^{n} \mathcal{D}_{i j, e f f}^{-1}\left(x_{i} \vec{J}_{j}^{D}-x_{j} \vec{J}_{i}^{D}\right)-D_{i K}^{-1} \vec{J}_{i}^{D}\right)=\sum_{i=1}^{n} \frac{\nabla P_{i}}{\mathcal{R} T}
$$

and, after simplification :

$$
\sum_{i=1}^{n} D_{i K}^{-1} \vec{J}_{i}^{D}=-\frac{\nabla P}{\mathcal{R} T}
$$

This relation is known as Graham's law of effusion. Introducing the bifurcation, eqs. (10) and (15) are rewritten :

$$
\begin{gathered}
\sum_{j \neq i}^{n} \eta_{b}\left(x_{i} \vec{J}_{j}^{D}-x_{j} \vec{J}_{i}^{D}\right) F_{i} F_{j}-\eta_{K} \vec{J}_{i}^{D} F_{i} F_{K}=\epsilon D^{r e f} \frac{\nabla P_{i}}{\mathcal{R} T} \\
\sum_{i=1}^{n} \vec{J}_{i}^{D} F_{i} F_{K}=-\epsilon \eta_{K}^{-1} D^{r e f} \frac{\nabla P}{\mathcal{R} T}
\end{gathered}
$$


Then the following null term is introduced in (16) :

$$
\eta_{b}\left(x_{i} \vec{J}_{i}^{D} F_{i} F_{i}-x_{i} \vec{J}_{i}^{D} F_{i} F_{i}\right)
$$

Eq. (16) now reads :

$$
\eta_{b}\left(x_{i} F_{i} \sum_{j=1}^{n} \vec{J}_{j}^{D} F_{j}-\vec{J}_{i}^{D} F_{i} \sum_{j=1}^{n} x_{j} F_{j}\right)-\eta_{K} \vec{J}_{i}^{D} F_{i} F_{K}=\epsilon D^{r e f} \frac{\nabla P_{i}}{\mathcal{R} T}
$$

Combining this last equation with eq. (17) yields :

$$
-\frac{\epsilon D^{r e f}}{\mathcal{R} T}\left(\nabla P_{i}-\eta_{b} \eta_{K}^{-1} \frac{x_{i} F_{i}}{F_{K}} \nabla P\right)=\left(\eta_{b} \sum_{j=1}^{n} x_{j} F_{j}+\eta_{K} F_{K}\right) \vec{J}_{i}^{D} F_{i}
$$

Using mass fluxes instead of mole fluxes, one obtains finally a Fickian form :

$$
\vec{j}_{i}^{D}=-\sum_{j=1}^{n} D_{i j} \nabla P_{j}
$$

where the porous-medium multicomponent diffusion coefficients $D_{i j}$ read :

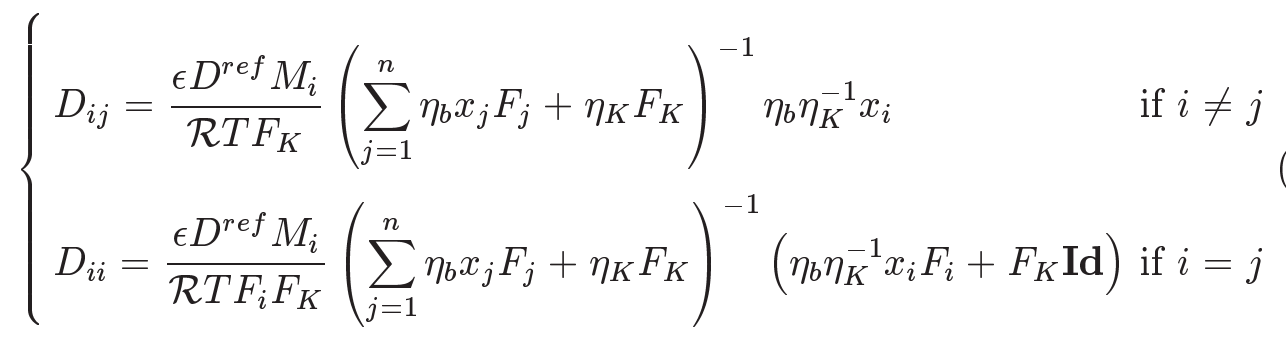

\subsection{Determination of the Knudsen diffusion factor}

The Knudsen diffusion factor $F_{K}$ may be directly determined from the inversion of (13) :

$$
F_{K}=\frac{D^{r e f}}{F_{i} D_{i}^{K}}
$$


Whatever the species $i$, the result for $F_{K}$ should be the same. This is only approximately the case, since the $F_{i}$ factors are only approximately proportional to $M_{i}^{1 / 2}$. Fig. 5 is a plot of $F_{K}$ from eq. (23) for the preceding 15 -species set. It is clearly seen from this figure that the discrepancy from the average is larger for the lightest gaseous species, and mainly for hydrogen. Alternatively to the choice of a simple average, the $F_{K}$ factor may be determined by the aforementioned simplex method, altogether with the $F_{i}$ factors.

Knudsen diffusion depends on temperature and pressure in a distinct way than ordinary diffusion : the former varies as $T^{1 / 2}$ while the latter varies as $T^{3 / 2} P^{-1}$ or more (actually, as $T^{1.75} P^{-1}$ according to Fuller's model for binary diffusion coefficients (Reid et al. (1987))). A consequence of this is that if $D^{\text {ref }}$ carries the usual $T^{1.75} P^{-1}$ dependence, then the Knudsen bifurcation factor $F_{K}$ has to be an explicit function of temperature and pressure, while the $F_{i}$ are not:

$$
F_{K}=F_{K}^{0}\left(\frac{T}{T^{0}}\right)^{1.25}\left(\frac{P^{0}}{P}\right)
$$

\subsection{Computational accuracy}

The multicomponent diffusion matrices obtained through this bifurcation method have been compared to the standard Dusty-Gas Model values in the 15-species test case. Very acceptable mean errors are obtained (about 1\%), but the maximal error is important for the lightest species (up to 30\%). This could be expected from the results of the preceding section ; however, since one extra 
hypothesis is made with regard to Bartlett's original model, an interesting fact is that the mean error is lower in the porous medium case.

Figs. 7 and 6 are plots of the mean error as a function of temperature and pore diameter. Again, it appears that the temperature has only a small effect on the results. On the other hand, the pore diameter has a stronger influence : the larger the pores, the larger the mean error, but the smaller the maximal error. To interpret this fact, recall that the maximal error is due to the lightest gas species, for which an a priori evaluation of $F_{K}$ would be substantially larger than the average (fig. 5). Hence, this error (due to the extra hypothesis) becomes more and more visible as the pore radius decreases.

\section{Conclusion}

An extension of the bifurcation method presented by Bartlett et al. (1968) to the Dusty-Gas Model (Mason and Malinauskas (1983)) has been presented and tested. It provides an explicit expression for the diffusive multicomponent fluxes in porous media which retains Graham's law of effusion as a constraint. The approximated coefficients exhibit the same degree of precision than when no porous medium is present, that is :

- The mean error is of the same order of magnitude, and even somewhat lower.

- The most important errors also concern the lightest gas species ; they are 
a little more important.

- Tests performed in the absence of very light gas species have proved very satisfactory accuracy on all species.

The limits of combining the bifurcation method and the Dusty-Gas Model are only the independent limits of both models ; so, inside them, it appears as a valuable computational speed-up for problems dealing with mass transport of large chemical systems in porous media like char combustion, pyrocarbon infiltration, etc ...

\section{Acknowledgements}

The authors are indebted to SEP, divison de SNECMA, for a Ph.D. grant to C.D.. They also wish to thank Ph. Le Helley (SEP-SNECMA) and G. Duffa (CEA) for helpful discussions. 


\section{References}

Bartlett, E. P., Kendall, R. M., and Rindal, R., An analysis of the coupled chemically reacting boundary layer and charring ablator. Part IV : A unified approximation for mixture transport properties for multicomponent boundary layer applications, Technical Report CR-1063, NASA, (1968).

Bird, R. B., Stewart, W. E., and Lightfoot, E. N., Transport Phenomena, John Wiley, New York City, NY, USA, 1960.

Giovangigli, V., Modélisation de la Combustion, CNRS, Paris, France, Images des Mathématiques, 1996.

Mason, E. A. and Malinauskas, A. P., Gas transport in porous media: the Dusty-Gas model, Chemical engineering monographs, Elsevier, Amsterdam, The Netherlands, 1983.

Ofori, J. Y. and Sotirchos, S. V., Multidimensional modeling of chemical vapor infiltration: Application to isobaric CVI, Ind. Eng. Chem. Res. 36 (1997), 357-367.

Reid, R. C., Prausnitz, J. M., and Poling, B. E., The properties of gases and liquids, Mc Graw Hill Book Company, New York City, NY, USA, $4^{\text {th }}$ edition, 1987.

Svehla, R. A., Estimated viscosities and thermal conductivities of gases at high temperatures, Technical Report R-132, NASA, 1962. 


\section{$6 \quad$ Figures and tables}

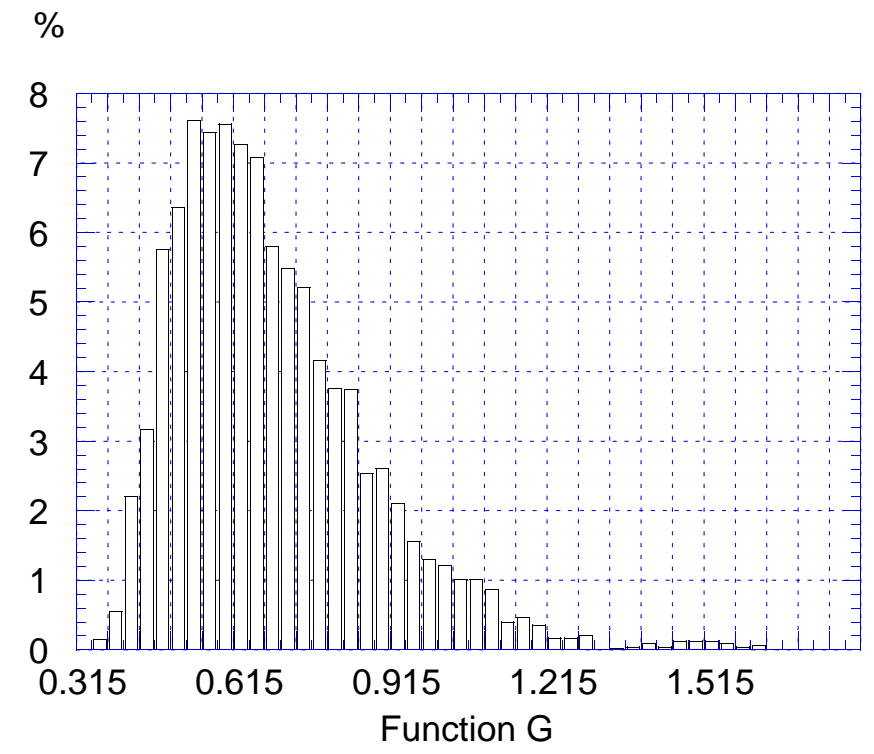

Fig. 1. Histogram of $G$ (see eq. (7) in text)

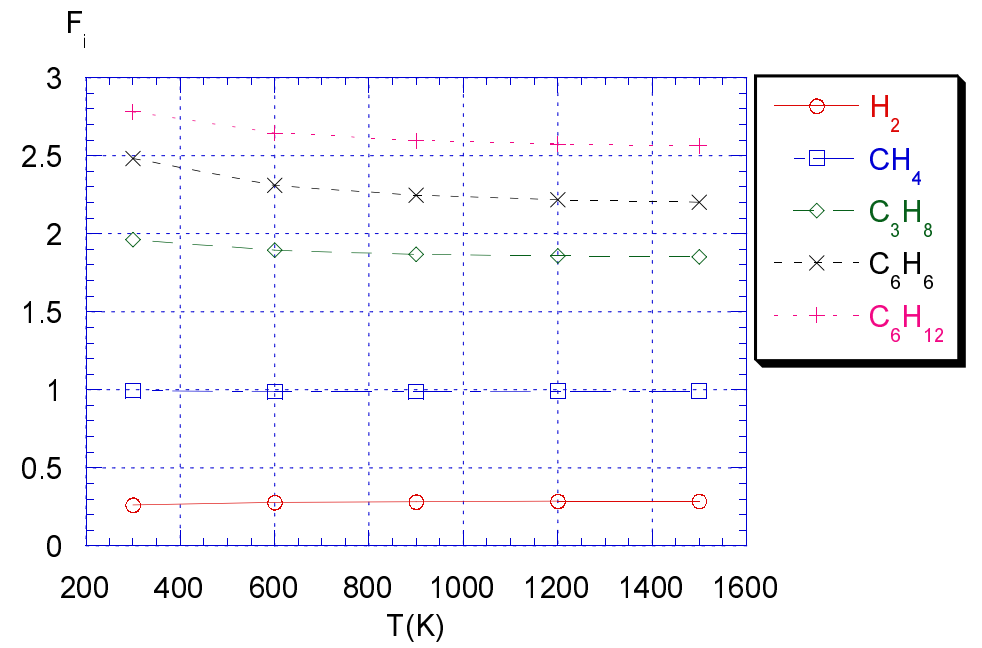

Fig. 2. Bifurcation coefficients $F_{i}$ vs. temperature. Chemical system of $5 \mathrm{C}$ - and H-containing gaseous species. 


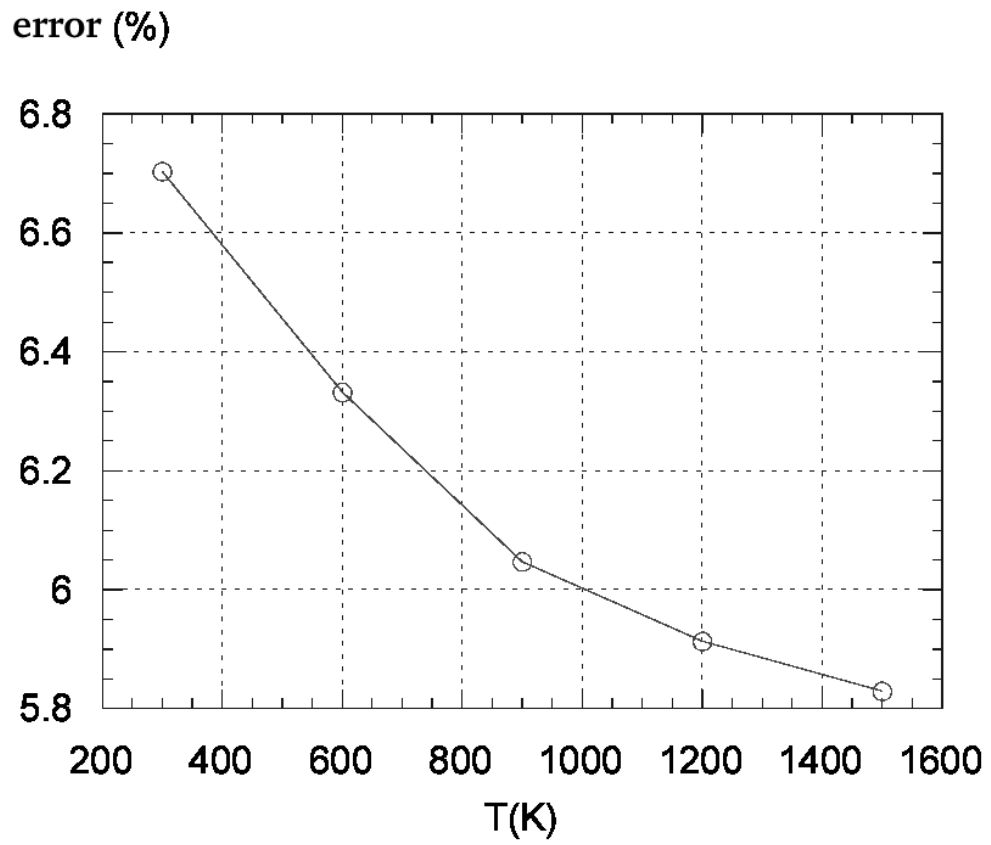

Fig. 3. Mean error on the diffusion coefficients vs. temperature. Chemical system of $5 \mathrm{C}$ - and H-containing gaseous species. 


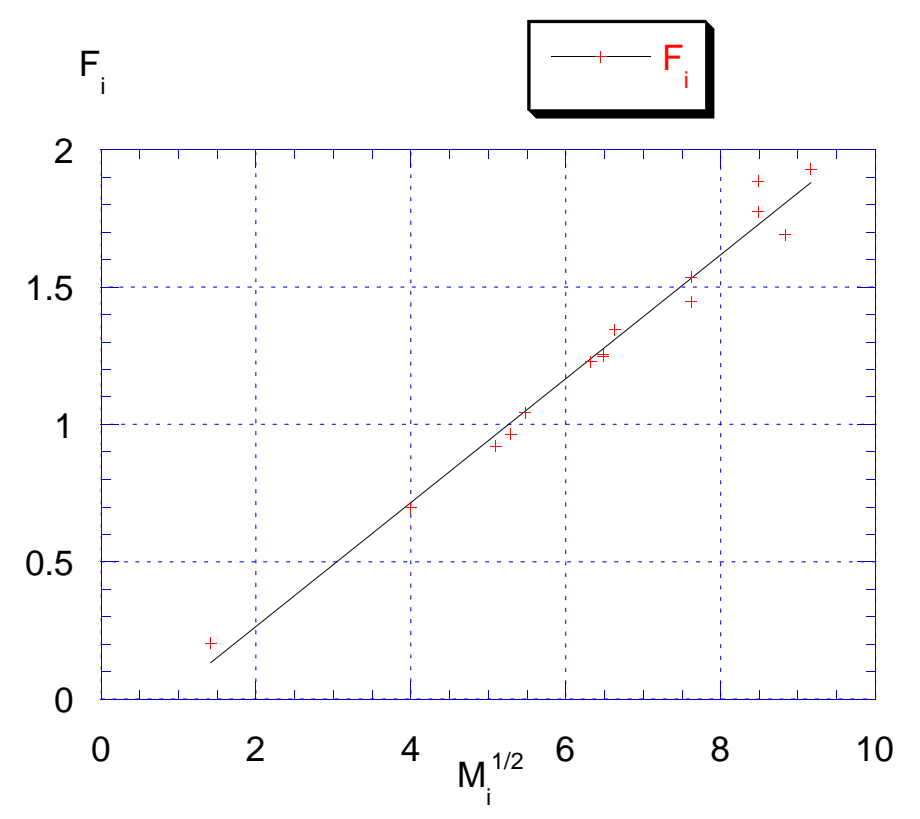

Fig. 4. Plot of diffusion factors vs. the square root of the molar mass.

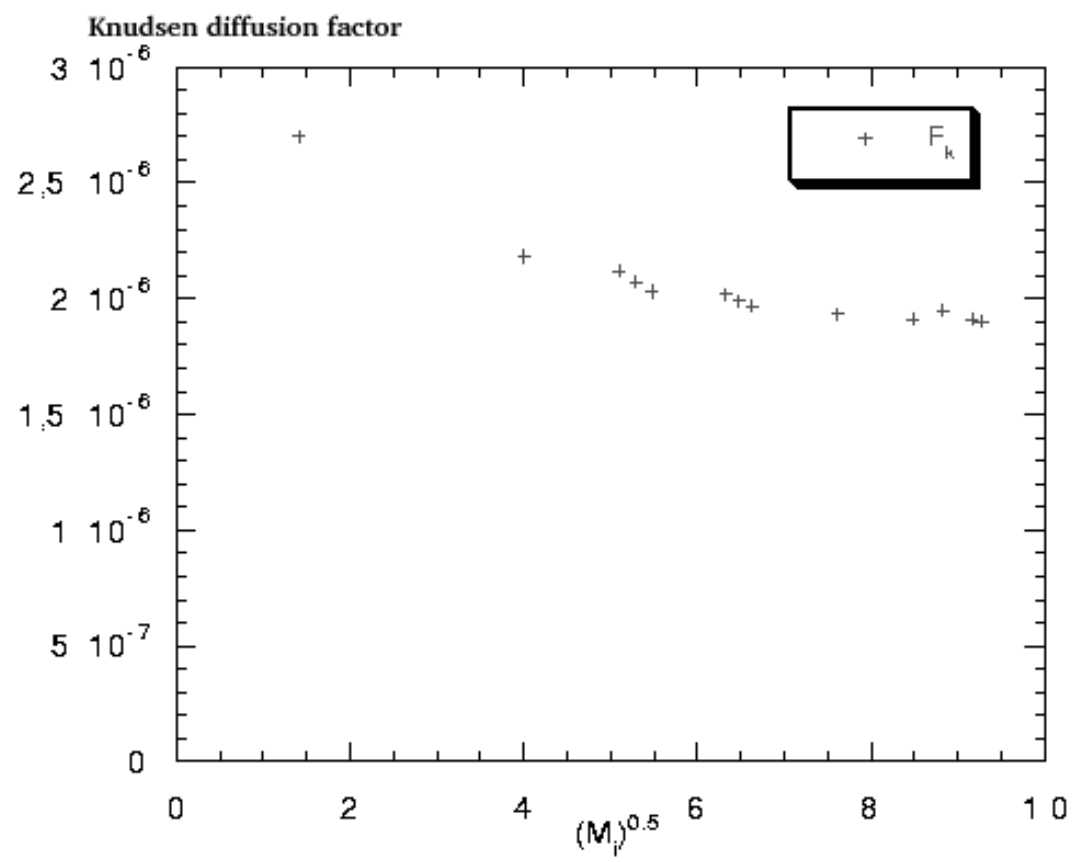

Fig. 5. Plot of the Knudsen diffusion factor from eq. (23) vs. the square root of the molar mass. 


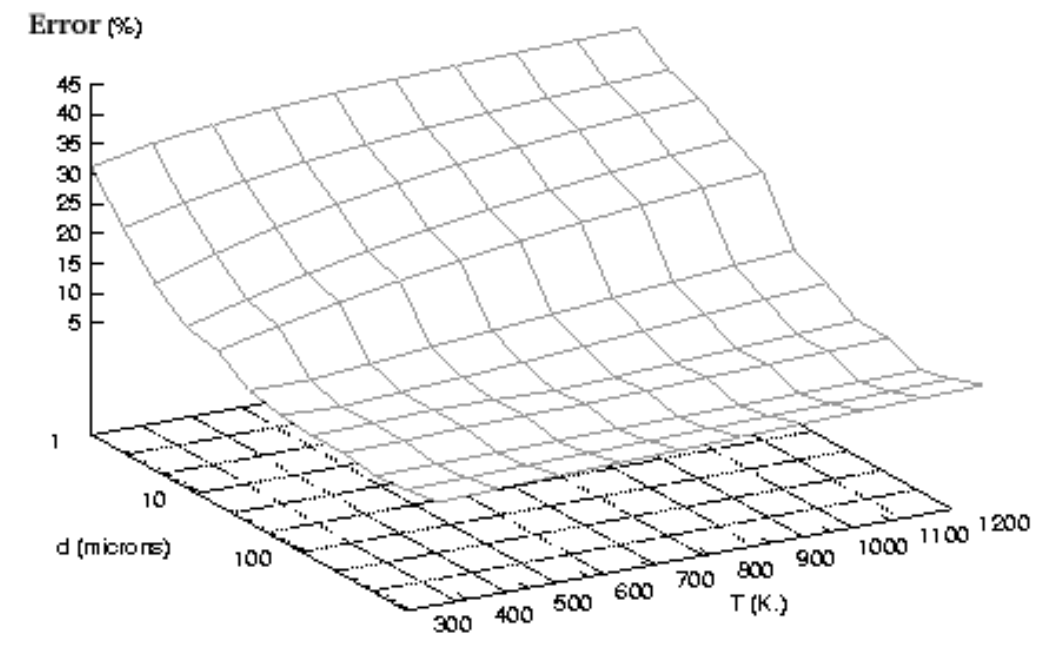

Fig. 6. Maximal error in $\%$ on the multicomponent diffusion matrix with respect to the largest diffusion coefficient.

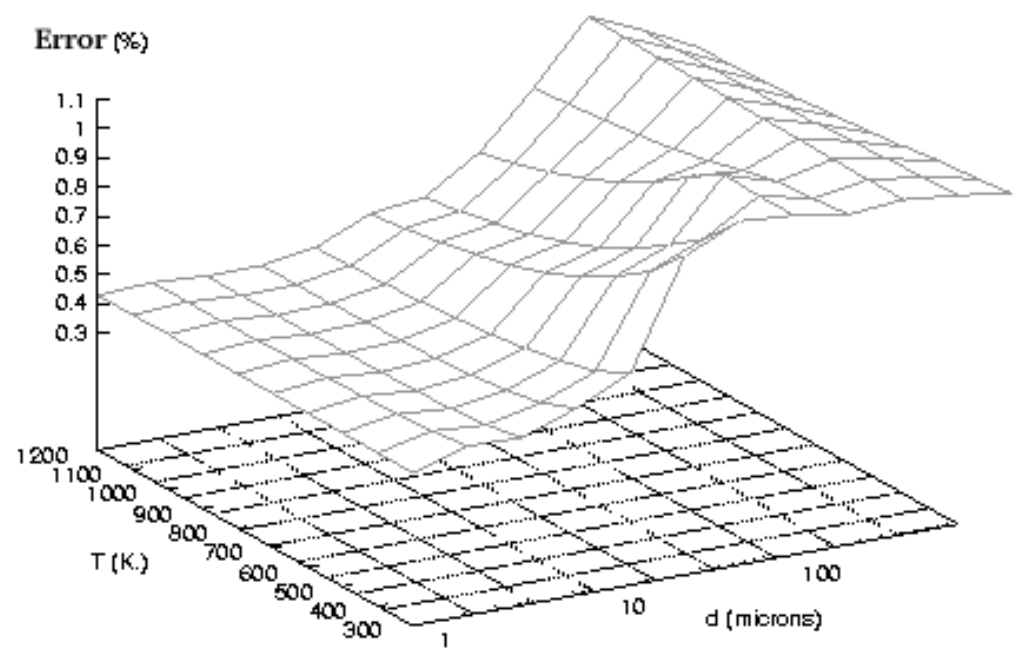

Fig. 7. Mean error in $\%$ on the multicomponent diffusion matrix with respect to the largest diffusion coefficient. 